\title{
ENFERMEDAD DE PAGET DE LA MAMA, SIN CARCINOMA DUCTAL ASOCIADO: REPORTE DE CASO Y REVISIÓN DE LA LITERATURA
}

\section{Paget's disease of the breast without associated ductal carinoma: Case report and review of the} literature

\author{
Liseth Johana Matamoros-Parra, $\mathrm{MD}^{1}$; Miguel Andrés Vertel-Velásquez, $\mathrm{MD}^{2}$; \\ Gloria Eugenia Camargo-Villalba, $M^{3}$
}

Recibido: mayo 25/18 - Aceptado: marzo 21/19

\section{RESUMEN}

Objetivo: presentar un caso de enfermedad de Paget (EP) de la mama, sin carcinoma ductal asociado, y realizar una revisión de literatura respecto a su diagnóstico y tratamiento.

Materiales y métodos: mujer de 59 años, con lesión pruriginosa, eritematosa y ulcerada crónica, en pezón izquierdo, de 2 años de evolución, quien recibió inicialmente manejo dermatológico con corticoides tópicos sin mejoría clínica, que acude finalmente a una institución de atención primaria en Tunja (Colombia) para su diagnóstico, el cual se realizó por biopsia mediante inmunohistoquímica y posterior manejo por cuadrantectomía central con patología posquirúrgica negativa para malignidad, sin recurrencia al año de seguimiento. Se realizó una búsqueda en las bases de datos Medline vía

1 Médica, Universidad de Boyacá, Programa de Medicina. Docente tiempo completo. Grupo de investigación Hygea. Tunja (Colombia). ljmatamoros@uniboyaca.edu.co

2 Médico, Universidad de Boyacá, Programa de Medicina. Grupo de investigación Hygea. Tunja (Colombia).

3 Médica Ginecoobstetra; mastóloga. Docente titular, Universidad de Boyacá, Programa de Medicina. Grupo de investigación Hygea. Tunja (Colombia).
PubMed, SciELO, ProQuest, ScienceDirect y Google Académico con los términos "Paget's Disease", "Breast Neoplasms", "Mammography" "Ultrasonography Mammary", "Biopsy” y "Mastectomy", rastreando artículos de revisión bibliográfica, editoriales, reportes y series de casos clínicos en inglés y español, sin límite de tiempo.

Resultados: se incluyeron 11 publicaciones correspondientes a 5 reportes de caso, 3 series de casos, 1 revisión de la literatura a partir de un caso clínico, y 2 cartas al editor que describieron casos relacionados con enfermedad de Paget del pezón sin carcinoma asociado. El diagnóstico se fundamentó en la sospecha clínica de la enfermedad con la posterior realización de biopsia de la lesión; la inmunohistoquímica fue útil en el diagnóstico diferencial. El tratamiento varió desde mastectomía hasta resección de complejo areola-pezón. La radioterapia también ha sido utilizada.

Conclusiones: el diagnóstico de la enfermedad de Paget sin carcinoma ductal asociado se fundamenta en una sospecha clínica temprana y en el estudio histopatológico del complejo areola-pezón. No hay consenso sobre el manejo quirúrgico de esta le- 
sión. Se requieren estudios de seguimiento de este subgrupo de pacientes tratado con radioterapia o cirugía conservadora.

Palabras clave: enfermedad de Paget mamaria, neoplasias de la mama, mamografía, ultrasonografía mamaria, biopsia, mastectomía.

\section{ABSTRACT}

Objective: To present one case of Paget's disease (PD) of the breast without associated ductal carcinoma, and to conduct a review of the literature on its diagnosis and treatment.

Materials and methods: A 59-year-old woman with a two-year history of a chronic erythematous, ulcerated, itchy lesion in the left nipple, initially treated dermatologically with topical steroids with no improvement, finally visits a primary healthcare institution in the city of Tunja, Colombia for diagnosis. A biopsy and histochemical testing were performed to make the diagnosis and the lesion was then treated with central quadrantectomy. The post-operative pathology testing was negative for malignancy, without recurrence at one-year followup. A search was conducted in the PubMed, SciELO, ProQuest, ScienceDirect and Google Scholar databases using the terms "Paget's Disease," "Breast Neoplasms," "Mammography," "Ultrasonography," Mammary," "Biopsy," and "Mastectomy," tracking bibliographic review articles, editorials, reports and clinical case series in English and Spanish, without time limitation.

Results: Overall, 11 publications were included consisting of 5 case reports, 3 case series, 1 review of the literature based on a clinical case, and 2 letters to the editor describing cases related to Paget's disease of the nipple with no associated carcinoma. The diagnosis was based on the clinical suspicion of the disease and a biopsy of the lesion. Histochemistry was useful for the differential diagnosis: treatment options ranged from mastectomy to nipple-areolar complex resection. Radiotherapy has also been used.
Conclusions: The diagnosis of Paget's disease with no associated ductal carcinoma is based on early clinical suspicion and histopathology of the nippleareolar complex. There is no consensus regarding surgical management of this lesion. Follow-up studies of this subgroup of patients treated with radiotherapy or conservative surgery are required.

Key words: Paget's disease of the breast, breast neoplasms, mammography, mammary ultrasound, biopsy, mastectomy.

\section{INTRODUCCIÓN}

La enfermedad de Paget (EP) de la mama constituye una patología localizada en la epidermis del pezón que comúnmente se encuentra asociada a carcinoma ductal subyacente $(1,2)$. Fue descrita por primera vez por Velpeau en 1856, quien identificó las características clínicas de la enfermedad (3). Posteriormente, en 1874 sir James Paget, un cirujano y fisiólogo británico, realizó una descripción más detallada de la enfermedad, por medio del reporte de 15 casos de mujeres con ulceración crónica de la piel del complejo areola-pezón, que tuvieron subsecuentemente un cáncer invasivo de glándula mamaria dos años después (4).

La enfermedad de Paget es una forma poco común de presentación del cáncer mamario, representa el 1-3\% de todas las neoplasias malignas del seno a nivel mundial $(5,6)$. Se ha reportado su aparición en mujeres con edades comprendidas entre los 27 y 88 años, con una mediana de 54 años (7). Aunque en el hombre la enfermedad es más rara, representa el 1,4\% de los cánceres mamarios, con una media de diagnóstico de 68 años (8).

En la actualidad existen dos teorías que intentan explicar la histogénesis patológica de las células de Paget: a) la teoría epidermotrópica considera que las células de Paget se originan a partir de un adenocarcinoma ductal mamario preexistente, que migra por medio de las membranas basales de los conductos galactóforos subyacentes hasta la epidermis del pezón (9); y b) la teoría de la trans- 
formación in situ que postula que la célula de Paget es un queratinocito epidérmico del pezón que ha sido transformado en maligno y desarrolla un carcinoma epidermoide independiente de cualquier carcinoma ductal subyacente (10), lo cual podría explicar los casos reportados de la enfermedad sin carcinoma asociado.

Según la Organización Mundial de la Salud (OMS), dicha patología se caracteriza por la infiltración neoplásica del epitelio escamoso del pezón a causa de células epiteliales glandulares malignas, conocidas como células de Paget (11). Dichas células se caracterizan por ser grandes, redondas u ovoides - de adenocarcinoma-, intraepidérmicas, con citoplasma claro y abundante, núcleos pleomórficos, hipercromáticos, aumentados de tamaño, y nucléolos prominentes $(12,13)$. Estas estructuras nunca hacen contacto con la membrana basal y pueden afectar el epitelio de los folículos sin incluir a la dermis; por otra parte, las células epidérmicas que rodean a las células de Paget sufren atrofia por compresión, y la dermis evidencia cambios como vasodilatación, edema y un infiltrado inflamatorio crónico compuesto por linfocitos, neutrófilos, eosinófilos y mastocitos, encargados de desarrollar la apariencia clínica característica de una dermatitis eccematosa y ulcerativa del pezón (14), por lo que el diagnóstico diferencial es a menudo la dermatitis atópica o la micosis de la mama (15). Por otra parte, es frecuente, como se mencionó, que la lesión se asocie a carcinomas ductales in situ o invasivos subyacentes (16).

Es importante que el ginecólogo piense en esta patología al tratar lesiones eccematosas del pezón en la quinta y sexta décadas de la vida, de manera que el manejo de las anteriores condiciones retrase lo menos posible el diagnóstico de la patología (17), ya que un diagnóstico oportuno mejora el pronóstico de la enfermedad, como es el caso de enfermedad de Paget sin carcinoma ductal subyacente.

El objetivo de este estudio es reportar el caso de una paciente con enfermedad de Paget de la mama, sin carcinoma ductal asociado, a quien se le realizó cuadrantectomía central como tratamiento definitivo, y hacer una revisión de la literatura con respecto al diagnóstico y tratamiento de esta entidad.

\section{PRESENTACIÓN DEL CASO}

Mujer de 59 años, raza mestiza, quien llega remitida por medicina general en el año 2015 al programa de “detección precoz de enfermedades de la mama", a la institución pública de primer nivel de atención, ESE Santiago de Tunja, sede La Fuente, institución que atiende población del régimen de aseguramiento subsidiado en el Sistema General de Seguridad Social en Salud (SGSSS) de Colombia. Tunja es una ciudad ubicada en la región centro-oriental del país. La paciente informaba cuadro clínico de dos años de evolución consistente en lesión pruriginosa del complejo areola-pezón izquierdo, ulcerativa, acompañada de secreción serosanguinolenta, automedicada inicialmente de forma tópica y posteriormente medicada con corticoides tópicos, con persistencia del cuadro clínico. Como antecedentes refería menarquia y telarquia a los 12 años, ciclos 30 x 5; gesta 2, cesáreas 1, abortos 1; edad de última menstruación: 49 años, edad del último parto: 26 años. Al examen físico se observa paciente en buenas condiciones generales, peso: $72 \mathrm{~kg}$, talla: 1,61 m, índice de masa corporal (IMC): 27,93, tensión arterial (TA): 125/ $80 \mathrm{~mm} / \mathrm{Hg}$, frecuencia cardiaca (FC): 70 lat/min, frecuencia respiratoria (FR): 18 $\mathrm{resp} / \mathrm{min}$. Al examen de mama se observó asimetría en el tamaño y la coloración de los pezones, con el pezón izquierdo de mayor tamaño y coloración eritematosa. Presentaba lesión ulcerada en pezón y areola izquierdos, con costras melicéricas y un área lisa e hipopigmentada en la región superior de la areola (figura 1). No se identificaron masas palpables en mamas ni adenopatías axilares, supra o infraclaviculares. Complejo areola-pezón derecho de características normales.

Con impresión diagnóstica de enfermedad de Paget de la mama se considera prudente solicitar estudios imagenológicos de mamografía bilateral y ultrasonografía mamaria bilateral. La mamografía 


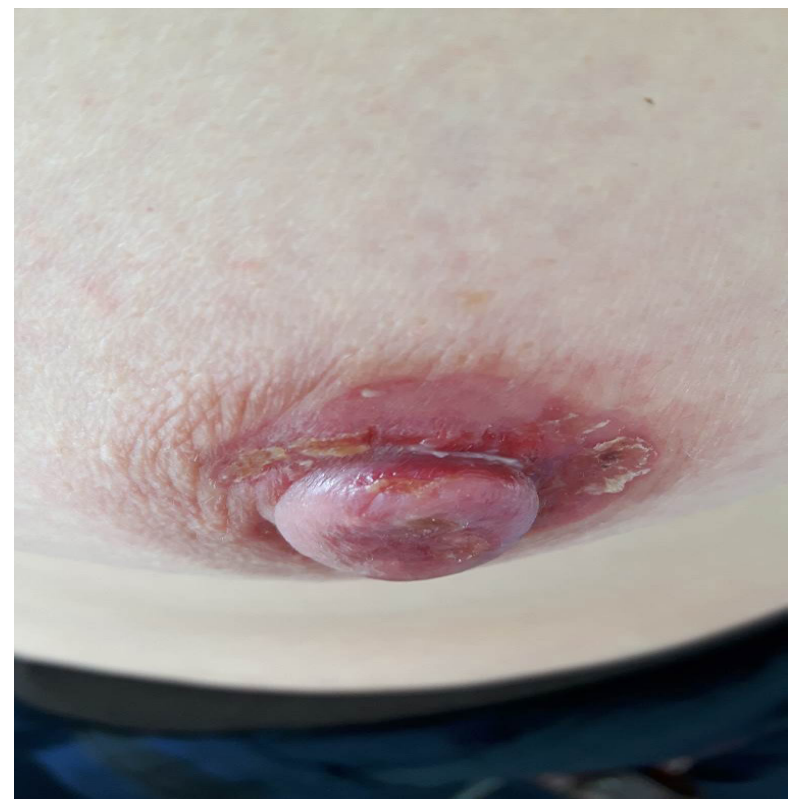

Figura 1. Lesión en el complejo areola-pezón izquierdo, con áreas ulceradas y costras melicéricas. Área lisa e hipopigmentada en región superior de la areola

evidenció mamas en forma y tamaño normal, parénquima mamario con moderada transformación adiposa y restos glandulares levemente densos, no acordes con edad de la paciente; en región retroareolar izquierda se observaron tenues micro- calcificaciones irregulares, heterogéneas (figura 2). Tejido vascular normal, planos posteriores libres, se observaban ganglios axilares bilaterales. El ultrasonido mamario realizado con transductor lineal de 10 MHZ evidenció edema de piel en el complejo areola-pezón izquierdo que medía 4,5 mm de diámetro anteroposterior, con pérdida de la continuidad de la línea hiperecogénica posterior de la piel (figura 3). El parénquima mamario muestra una distribución regular de ecos, sin imágenes nodulares sólidas ni líquidas. En las regiones axilares no se observaron imágenes de significado patológico.

Teniendo en cuenta la edad de la paciente, la sintomatología crónica de prurito, las características clínicas de la lesión, la falta de respuesta al tratamiento instaurado, las microcalcificaciones evidenciadas en región retroareolar izquierda en la mamografía y el edema de la piel del complejo areola-pezón observado en ultrasonido, se presenta sospecha clínica e imagenológica de enfermedad de Paget de la mama, por lo cual se solicita biopsia. El resultado de la patología reportó lesión intraepidérmica epitelial atípica en la piel de la areola izquierda que planteaba enfermedad de Paget frente

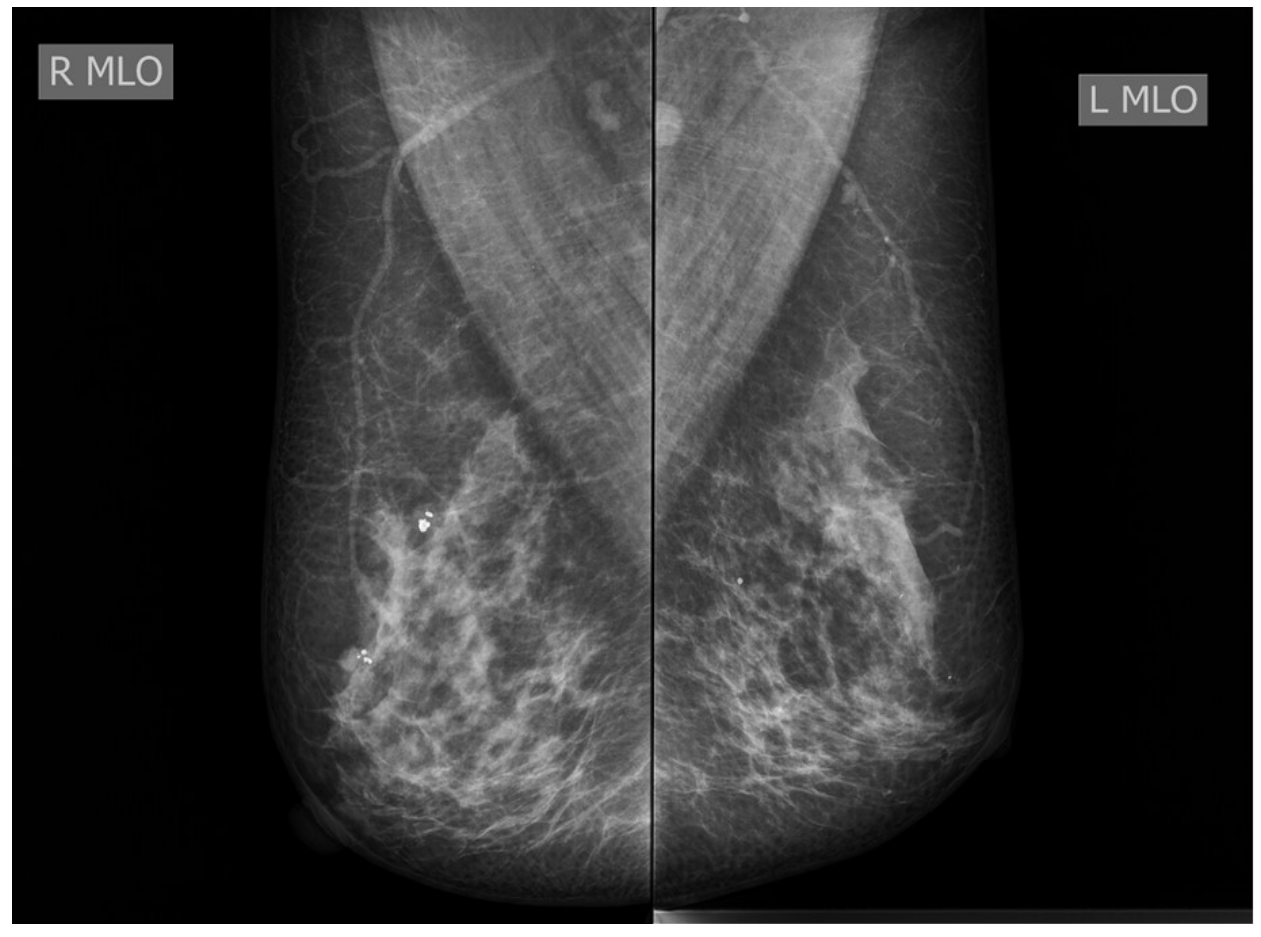

Figura 2. Mamografía: tenues microcalcificaciones irregulares y heterógenas en región retroareolar izquierda 


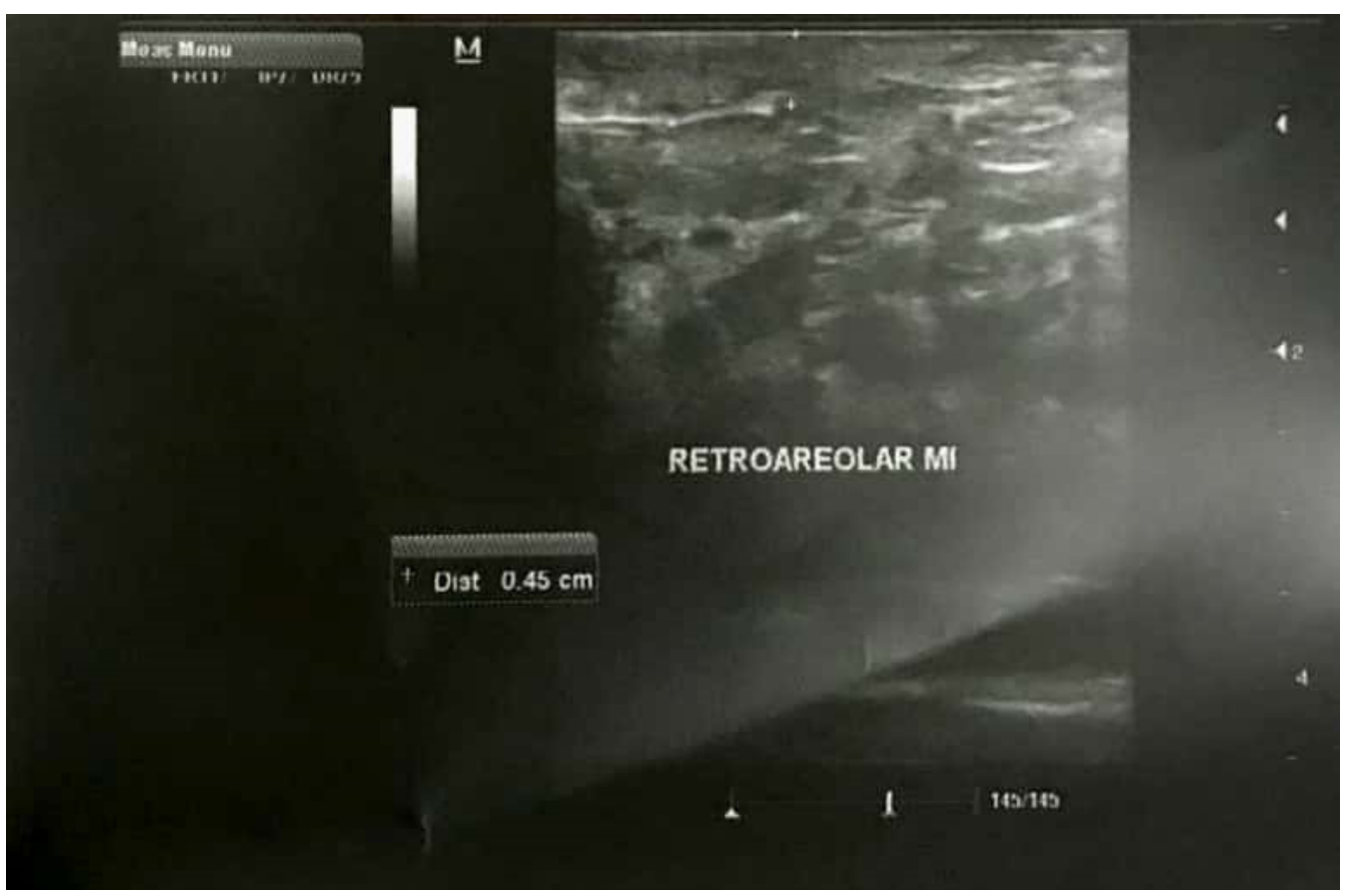

Figura 3. Ecografía mamaria: diámetro anteroposterior del complejo areola-pezón izquierdo aumentado $(4,5 \mathrm{~mm})$ y pérdida de la continuidad de la línea hiperecogénica posterior de la piel

a enfermedad de Bowen, motivo por el cual se realizó estudio de inmunohistoquímica en el que dermopatología identificó células positivas para CK7 y negativas para CK20 (figura 4), lo que confirmó la enfermedad de Paget del complejo areola-pezón izquierdo.

Con diagnóstico de enfermedad de Paget fue remitida a oncocirugía de mama y tejidos blandos para su manejo quirúrgico en mayo de 2017, donde se practicó cuadrantectomía central del seno izquierdo, sin complicaciones. El informe de patología de la muestra obtenida reportó papilomas intraductales con metaplasia apocrina sin atipia nuclear, y cambios de ectasia con infiltrados plasmocitarios secundarios. Líneas de sección negativas para tumor, lo cual descarta la presencia de carcinoma subyacente y elimina la necesidad de las sesiones de radioterapia propuestas inicialmente por el médico tratante.

$\mathrm{Al}$ año de control la paciente ha tenido una evolución clínica posquirúrgica satisfactoria, la mamografía de control 10 meses posoperatorio no mostró hallazgos patológicos (figura 5). No ha presentado complicaciones y se encuentra asintomática.

\section{MATERIALES Y MÉTODOS}

Se realizó una búsqueda de información por medio de bases de datos como Medline vía PubMed, SciELO, ProQuest, ScienceDirect y Google académico con los términos de búsqueda MeSH: "Paget's Disease", "Breast Neoplasms", "Mammography"

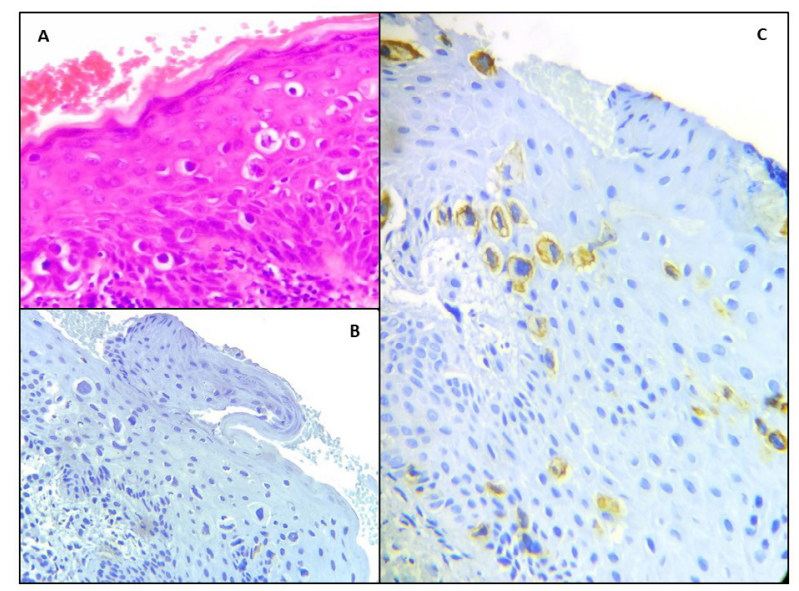

Figura 4. A. Tinción HE: se observa epidermis con infiltración por células con marcadas atipias nucleares, anaplásicas. B. CK7: Estudio de inmunohistoquímica con CK7 positivo en las células tumorales. C. CK20: estudio de inmunohistiquímica con CK20 negativo para malignidad 


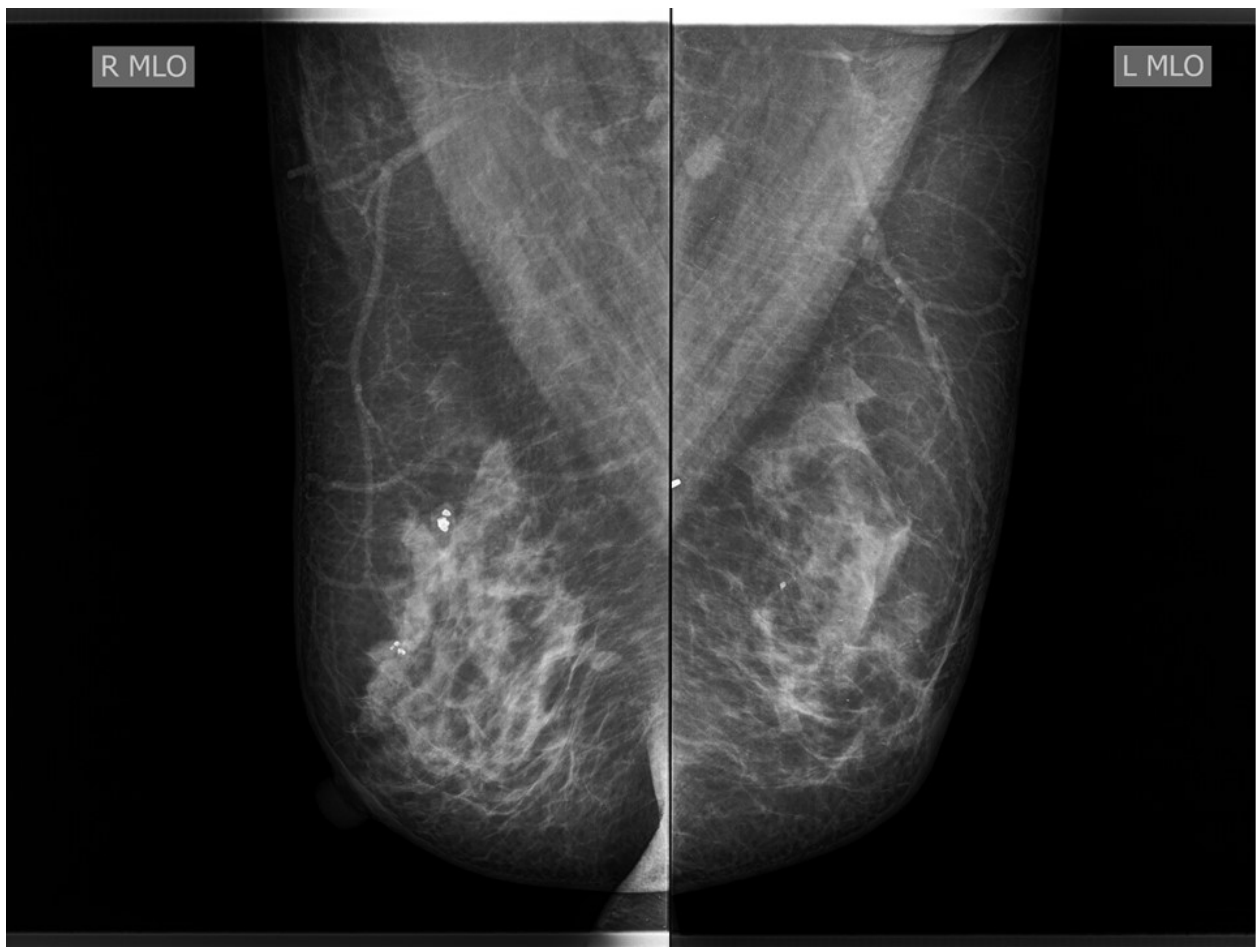

Figura 5. Mamografía posquirúrgica: ausencia de complejo areola-pezón izquierdo

"Ultrasonography Mammary", "Biopsy” y "Mastectomy”, en artículos de revisión bibliográfica, reportes y series de casos clínicos y cartas al editor que describieran información relacionada con enfermedad de Paget de la mama, sin carcinoma asociado, en inglés y español. La búsqueda no se limitó a un periodo determinado de tiempo dada la rareza de la patología y la escasez de literatura referente al tema. Se realizó una selección por título y resumen por dos autores, y en caso de encontrar una discrepancia la elección se sometió a un tercer evaluador. Los datos extraídos de los artículos seleccionados incluyeron: identificación del artículo, edad, signos y síntomas, diagnóstico, tratamiento y resultados de la patología. Se excluyeron aquellas publicaciones en las cuales no se obtuvo acceso al texto completo.

Aspectos éticos. Se obtuvo consentimiento informado verbal y por escrito de la paciente para la publicación del caso y las fotografías, y se garantizó la confidencialidad de la información obtenida durante todo el proceso.

\section{RESULTADOS}

La búsqueda inicial mostró 42 títulos, de los cuales se seleccionaron 11 estudios que cumplieron con los criterios de búsqueda (figura 6). Se excluyeron 31 artículos por no tener ninguna referencia acerca de la enfermedad de Paget mamaria sin carcinoma asociado. Los artículos seleccionados corresponden a 5 reportes de casos (18-22), 3 series de casos (23-25), 1 revisión de la literatura a partir de un caso clínico (26) y 2 cartas al editor $(27,28)$. Todos ellos escritos en inglés, excepto uno (25), escrito en español.

Diagnóstico. En la literatura consultada se identificaron pacientes con edades comprendidas entre los 40 y los 80 años. En 5 de los casos reportados, la patología se manifestó como una lesión eritematosa y exfoliativa, de crecimiento progresivo en la piel del complejo areola-pezón $(18,20,22,23,26)$; en un caso se identificó una lesión tipo mácula hipopigmentada de borde eritematoso (21); otro presentaba una mácula hiperpigmentada y pruriginosa (28); dos casos se expresaron como masas mamarias, en uno 
Figura 6.

Esquema Prisma de selección de artículos

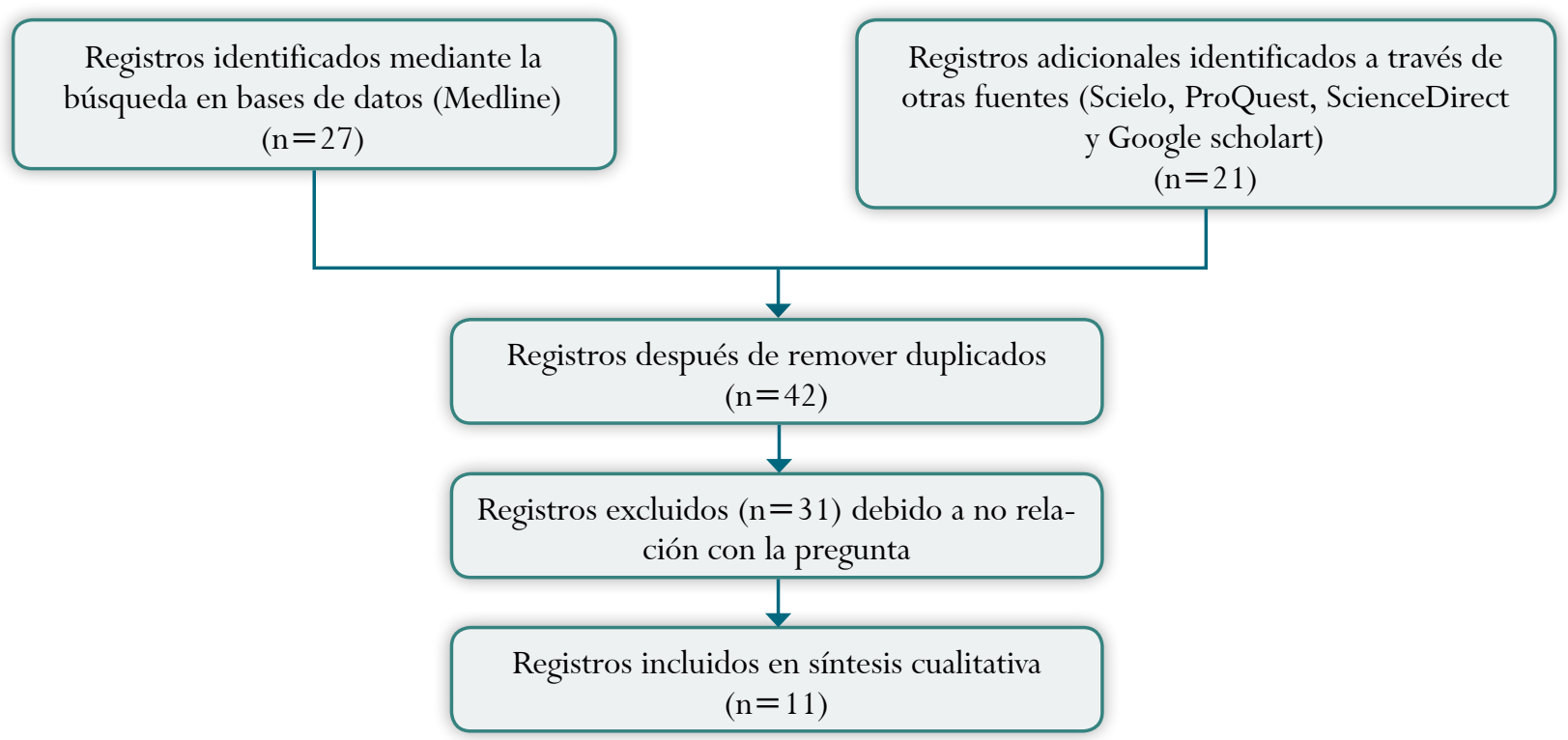

de ellos acompañada de eritema periareolar (19) y el otro sin cambios visibles en la piel o el pezón (27).

Adicionalmente, un estudio destacó el aspecto eccematoso como primer signo de la enfermedad en la mayoría de los pacientes (24), y otro artículo describió a la forma clínica ulcerativa como la más prevalente en la patología (25).

En todos los casos reportados, a excepción de uno (27), el diagnóstico definitivo de la enfermedad se realizó por medio de biopsia de la lesión, donde se identificaron las células de Paget características. En el caso adicional, el diagnóstico se hizo de forma incidental en una mujer de 47 años quien presentaba masas mamarias bilaterales palpables, sin antecedente de cambios en la piel o descargas por el pezón. La paciente tenía antecedente de dos familiares en primer grado y uno en segundo grado con cáncer de mama, motivo por el cual fue sometida a mastectomía bilateral profiláctica. El estudio de biopsia identificó fibroadenomas bilaterales y células de Paget en la piel del pezón izquierdo con infiltración a la dermis superficial, sin carcinoma ductal asociado (27).
Los estudios de inmunohistoquímica resultan útiles para realizar el diagnóstico diferencial de la enfermedad de Paget con otras patologías de comportamiento similar como el melanoma maligno y la enfermedad de Bowen (22). Dentro de los estudios seleccionados, tres reportaron células de Paget positivas para el antígeno carcinoembrionario (CEA) $(20,21,26)$, tres positivas para el antígeno de membrana epitelial (EMA) $(19,20,22)$ y dos positivas para citoqueratina 7 (CK7) $(26,28)$. Por su parte, en dos estudios las células fueron negativas para Melan A $(26,28)$. En el caso presentado el estudio de inmunohistoquímica reveló positividad para CK7 y negatividad para CK20, marcadores que permitieron confirmar el diagnóstico de enfermedad de Paget y descartar enfermedad de Bowen.

Tratamiento. En la actualidad, a pesar de que el manejo terapéutico estándar reportado en la literatura es la mastectomía simple, con o sin disección de ganglios linfáticos axilares (23), la cirugía conservadora se ha convertido en una alternativa para mujeres como la paciente del caso presentado, sin masas ni adenopatías palpables, debido a los resul- 
tados de supervivencia equiparables con el manejo radical, y la posibilidad de realizar una terapia menos invasiva (24).

En la literatura consultada el tratamiento de elección para la enfermedad de Paget sin carcinoma ductal subyacente fue la realización de mastectomía: simple en un caso (19), radical en otro (25), radical modificada en tres $(18,20,22)$, y mastectomía segmentaria con preservación del pezón en una paciente (26). En los demás casos se optó por intervenciones conservadoras: en dos casos se realizó escisión del complejo areola-pezón (21, 23), y en una paciente la cirugía se limitó a extirpar únicamente la lesión con margen de 2 mm (28). En todos estos casos la respuesta al tratamiento instaurado fue positiva y no se presentaron recidivas durante el seguimiento.

Lagios et al. (23) describieron cinco casos de enfermedad de Paget del pezón sin masas mamarias detectables clínicamente, dos de ellos sin carcinoma ductal subyacente, y los demás con carcinoma ductal in situ, muy limitado, que fueron manejados con cirugía conservadora. Cuatro pacientes se sometieron a escisión total del complejo areola-pezón afectado, a tres de ellos se les realizó biopsia dirigida mamográficamente o biopsia ciega de cuatro cuadrantes. La paciente restante se sometió a una resección parcial del complejo areolar del pezón con una muestra de ganglio linfático axilar. La media de seguimiento fue de 50 meses; la paciente manejada con escisión parcial del complejo areola-pezón tuvo recurrencia de la enfermedad 12 meses después del tratamiento, ninguno de los demás pacientes mostró recidivas de la enfermedad durante el periodo de seguimiento. El estudio determinó que las cirugías conservadoras pueden considerarse una opción de tratamiento en pacientes estrictamente seleccionados; sin embargo, no se descarta que puedan desarrollar lesiones invasivas posteriores y requerir manejo radical definitivo.

Por su parte, Fourquet et al. (24), en 1987, realizaron un estudio para evaluar la utilidad del manejo conservador con radioterapia en pacientes sin tumores de mama detectables. Incluyeron 20 pacientes con enfermedad de Paget confirmada, a una de ellas se le extirpó únicamente el pezón, y a otra el complejo areola-pezón completo, en los dos casos se adicionó radioterapia. Los 17 pacientes restantes fueron tratados únicamente con radioterapia. La mediana de seguimiento fue de 7,5 años. Ningún paciente murió de enfermedad mamaria. Tres pacientes tuvieron recurrencia en la mama tratada y fueron sometidos a mastectomía. Todas las recurrencias se localizaron en el pezón o la areola y se presentaron como enfermedad de Paget, sin carcinoma invasivo o intraductal asociado. El pronóstico de vida a 7 años fue de $81 \%$, y el resultado cosmético de las intervenciones fue en su mayoría bueno, concluyendo que la radioterapia podría ser una alternativa efectiva a las cirugías radicales en el manejo de pacientes con enfermedad de Paget sin tumores mamarios detectables.

Adicionalmente, en las pacientes que presentan carcinoma ductal invasivo el tratamiento debe incluir resección completa de la enfermedad subyacente combinada con escisión del complejo areola-pezón, seguido de radioterapia para el tejido mamario restante (24). Los manejos quirúrgicos amplios y la quimioterapia son reservados para lesiones invasivas y tumores extensos (23).

\section{CONCLUSIÓN}

El diagnóstico de la enfermedad de Paget sin carcinoma ductal asociado se fundamenta en la sospecha clínica de la enfermedad, con la posterior realización de biopsia de la lesión que confirma la patología, con el hallazgo de las células características; el tratamiento inicial sería la resección del complejo areola-pezón. Si se confirma que no hay carcinoma ductal asociado se podría considerar el seguimiento estricto.

\section{AGRADECIMIENTOS}

Agradecemos de manera especial a la doctora María Claudia Carrillo (dermatopatóloga) y a los doctores Juan Carlos Vega y Jesús Oropeza, patólogos adscri- 
tos a la empresa Famedit Lab Zell, Tunja, Boyacá, por las imágenes y los conceptos aportados.

\section{REFERENCIAS}

1. Valladares M, Conde T, Díaz N. Enfermedad de Paget. Variedad especial de cáncer mamario. Presentación de un caso. Medisur. 2012 [visitado 2018 abr 12];10:52225. Disponible en: http://www.medisur.sld.cu/index. $\mathrm{php} / \mathrm{medisur} /$ article/view/2301

2. Crignis GS, Abreu LD, Buçard AM, Barcaui CB. Polarized dermoscopy of mammary Paget disease. An Bras Dermatol. 2013;88:290-2. https://doi.org/10.1590/ S0365-05962013000200023

3. Gaurav A, Gupta V, Koul R, Dabas S, Sareen R, Geeta K, et al. Practical consensus recommendations for Paget's disease in breast cancer. South Asian J Cancer. 2018;7:83-6. https://doi.org/10.4103/sajc. sajc_107_18

4. Paget J. On disease of the mammary areola preceding cancer of the mammary gland. St Barts Hospital Rep. 1874 [visitado 2019 mar 10];10:87. Disponible en: https://onlinelibrary.wiley.com/doi/epdf/10.3322/ canjclin.21.5.303.

5. Betal D, Puri N, Roberts K, Kalra L, Rapisarda F, Bonomi R. Hyperpigmented Paget's disease of the nipple: A diagnostic dilemma. JRSM Short Rep. 2012;3: 1. https://doi.org/10.1258/shorts.2012.011165

6. Dominici LS, Lester S, Liao GS, Guo L, Specht M, Smith BL, et al. Current surgical approach to Paget's disease. Am J Surg. 2012;204:18-22. https://doi. org/10.1016/j.amjsurg.2011.07.010

7. Caliskan M, Gatti G, Sosnovskikh I, Rotmensz N, Botteri E, Musmeci S, et al Paget's disease of the breast: The experience of the European Institute of Oncology and review of the literature. Breast Cancer Res Treat. 2008;112(3):513-21. https://doi.org/10.1007/ s10549-007-9880-

8. Adams SJ, Kanthan R. Paget's disease of the male breast in the 21 st century: A systematic review. Breast. 2016;29:14-23. https://doi.org/10.1016/j.breast. 2016.06.015

9. Albarrán J, Tavares A, Saldivia F, Prince J, Ramos S, Gutiérrez Néstor, et al. Enfermedad de Paget de la mama. Reporte de 13 casos. Rev Venez Oncol.
2010 [citado 2018 abr 20];22:194-200. Disponible en: http://www.scielo.org.ve/scielo.php? script $={ }_{\text {sci }}$ arttext\&pid $=$ S0798-05822010000300008\&lng $=$ es.

10. Lopes LL, Lopes IM, Lopes LR, Enokihara MM, Michalany AO, Matsunaga N. Mammary and extramammary Paget's disease. An Bras Dermatol. 2015;90:225-31. https://doi.org/10.1590/abd1806-4841.20153189

11. Tavassoli FA, Devilee P (eds.). World Health Organization Classification of Tumours. Pathology and Genetics of Tumours of the Breast and Female Genital Organs. IARC Press, Lyon; 2003.

12. Muttarak M, Siriya B, Kongmebhol P, Chaiwun B, Sukhamwang N. Paget's disease of the breast: clinical, imaging and pathologic findings: A review of 16 patients. Biomed Imaging Interv J. 2011;7(2):e16. https://doi.org/ 10.2349/biij.7.2.e16.

13. Bas A, Rodríguez T, Arias LC. Enfermedad de Paget de la mama. Revista Electrónica Dr. Zoilo E. Marinello Vidaurreta. 2014 [citado 2018 abr 26];39. Disponible en: http://revzoilomarinello.sld.cu/index.php/zmv/ article/view/172/310

14. Casimiro L, Vilata JJ, Zaragoza V. Enfermedad de Paget mamaria. Med Cutan Iber Lat Am. 2008;36:3-12.

15. Vilchez M, Villarreal M, Garay I, Kurpis M, Ruiz A. Patología dermatológica de la mama. A propósito de 3 casos. Arch Argent Dermatol. 2011;61:137-41.

16. Duan X, Sneige N, Gullett AE, Prieto VG, Resetkova E, Andino LM, et al. Invasive Paget disease of the breast clinicopathologic study of an underrecognized entity in the breast. Am J Surg Pathol. 2012;36:1353-8. https:// doi.org/10.1097/PAS.0b013e318259ef7f.

17. Siponen E, Hukkinen K, Heikkla P, Joensuu H, Leidenius M. Surgical treatment in Paget's disease of the breast. Am J Surg. 2010;200:241-6. https://doi. org/10.1016/j.amjsurg.2009.07.044

18. Jones RE. Mammary Paget's disease without underlying carcinoma. Am J Dermatopathol. 1985;7(4):361-5. https://doi.org/10.1097/00000372198508000-00009

19. O'Sullivan ST, McGreal GT, Lyons A, Bruke L, Geoghegan JG, Brady MP. Paget's disease of the breast in a man without underlying breast carcinoma. J Clin Pathol. 1994;47(9):851-2. https://doi.org/10.1136/ jсp.47.9.851 
20. Mori O, Hachisuka H, Nakano S, Maeyama Y, Sasai Y. A case of mammary paget's disease without an underlying carcinoma: Microscopic analysis of the DNA content in Paget cells. J Clin Pathol. 1994;21:1605. https://doi.org/10.1111/j.1346-8138.1994. tb01714.x

21. Takeuchi T, Komatsuzaki M, Minesaki Y, Yokoi K, Kamide R, Niimura M, et al. Paget's disease arising near a male areola without an underlying carcinoma. J Clin Pathol. 1999; 26:248-52. https://doi. org/10.1111/j.1346-8138.1999.tb03466.x

22. Bansal S, Sahoo B, Agarwal P, Garg V, Rao S. A rare presentation of mammary Paget's disease involving the entire breast in the absence of any underlying ductal malignancy. Indian J Dermatol Venereol Leprol. 2013; 79:518-21. https://doi.org/10.4103/03786323.113085

23. Lagios MD, Westdahl PR, Rose MR, Concannon S. Paget's disease of the nipple alternative management in cases without or with minimal extent of underlying breasf carcinoma. Cancer. 1984;54:545-51. https://doi.org/ 10.1002/1097-0142(19840801)54:3<545::AIDNCR2820540327>3.0.CO;2-M

24. Fourquet A, Campana F, Vielh P, Schlienger P, Jullien D, Vilcog JR. Paget's disease of the nipple without de- tectable breast tumor: Conservative management with radiation therapy. Int J Radiation Oncology Biol Phys. 1987;13:1463-5. https://doi.org/10.1016/03603016(87)90311-7

25. Torres L, Puerto JA, Guerra E, Caballero N. Caracterización clínico-epidemiológica de la enfermedad de Paget de la mama. Estudio de 37 años. MediSur. 2017 [citado 2018 abr 26];15:277-81. Disponible en: https://www.redalyc.org/articulo. oa? id $=180046859011$

26. Broecker JS, Sewell CW, Shehata BM, Memis B, Adsay NV, Styblo TM. An atypical presentation of Paget's disease of the breast without nipple involvement: Case report and review of the literature. Pathol Res Pract. 2017;213:1454-56. https://doi.org/10.1016/j. prp.2017.06.002

27. Hanna M, Jaffer S, Bleiweiss IJ, Nayak A. Minimally invasive mammary Paget's disease without an underlying breast carcinoma. Virchows Arch. 2013;463:471-3. https://doi.org/10.1007/s00428-013-1446-9

28. Shiomi T, Ogata D, Iwata M, Arai E, Tsuchida T. Pigmented mammary Paget's disease without underlying carcinoma. J Dermatol. 2018;45: e321-e322. https:// doi.org/10.1111/1346-8138.14461 